\title{
Synthesis of some novel 1,2,4-triazole derivatives as potential antimicrobial agents
}

\author{
Mohamed Sayed Behalo *, Aly Abdelmaboud Aly, Ashraf Farouk Wasfy and Marwa Mohamed Rizk \\ Chemistry Department, Faculty of Science, Benha University, Benha, 13518, Egypt \\ *Corresponding author at: Chemistry Department, Faculty of Science, Benha University, Benha, 13518, Egypt. \\ Tel.: +2.010.1599607; fax: +2.013.3222578. E-mail address: mohamedbehalo@hotmail.com (M. Behalo).
}

\section{ARTICLE INFORMATION}

Received: 18 April 2012

Received in revised form: 05 June 2012

Accepted: 05 June 2012

Online: 30 June 2013

\section{KEYWORDS}

Quinoline

1,2,4-Triazole

Spectroscopy

Antifungal activities

Antibacterial activities

1,2,4-Triazolo[3,4-b] thiadiazole

\section{Introduction}

The triazole ring is a frequent partner in polycyclic heterocyclic systems of biological significance and industrial applications. Compounds containing a fused 1,2,4-triazole moiety have attracted attention in the past few years owing to their biological activity. A number of these class of compounds act as antibacterial [1-3], antifungal [4-6], anti-inflammatory [7], analgesic [8-10], antituberculosis [11] and anticonvulsant agents [12-14]. In addition, a large number of triazole derivatives have antiulcer $[15,16]$, antidepressant $[17,18]$, insecticidal [19], hypolipidemic [20], antidiabetic [21] and antimitotic activity [22].

Also, azetidin-2-ones of 3-pyridyl-4-amino-5-mercapto1,2,4-triazoles [23] and 4-( $N$-pyridylcarboxamido)-5-mercapto3-substitued 1,2,4-triazoles [24] have been reported to possess significant activity as antitumor and antiviral agents. On the other hand, quinoline derivatives have been proven as privileged core structures and confirmed in bioactive natural products and in various pharmaceutical agents. They are known for their antiplasmodial properties and have been used as the staring compounds for new antimalarial agents [25,26].

Prompted by the varied biological activities of triazole derivatives and in continuation of our efforts for the synthesis of biologically active heterocycles [27-29], the present work describes the synthesis of novel 1,2,4-triazole derivatives attached with quinoline moiety with the aim of enhancement of their biological activity.

\section{Experimental}

\subsection{Instrumentation}

Melting point of the synthesized compounds was determined in Perkin Elmer apparatus and is uncorrected. IR spectra were recorded using $\mathrm{KBr}$ pellets. ${ }^{1} \mathrm{H}$ NMR spectra were recorded on Mercury-300BB (NMR 200) in $\mathrm{CDCl}_{3}, \mathrm{DMSO}_{-} d_{6}$ as solvent. Tetramethylsilan (TMS) served as an internal reference and chemical shifts were expressed in $\delta$ (ppm). Mass spectra were recorded on GC-MS Shimadzu QP2010. All the microanalysis and antimicrobial activities were performed by the Micro analytical unit, Cairo University.

\subsection{Synthesis}

\subsubsection{2-(Quinolin-8-yloxy)acetohydrazide (1)}

The titled compound was prepared according to the method described in the literature [30]. A mixture of hydrazine hydrate $(0.01 \mathrm{~mol})$ and ethyl 2-(quinolin-8-yl-oxy)acetate $(0.01$ $\mathrm{mol})$ in ethanol $(30 \mathrm{~mL})$ was heated under reflux for 4 hours After cooling, the precipitated solid was collected and recrystallized from ethanol. Color: Yellow. Yield: 70\%. M.p.: 218-220 ${ }^{\circ}$ C. FT-IR (KBr, v, cm-1): 3322-3254 $\left(\mathrm{NH}_{2}, \mathrm{NH}\right), 1661$ $(\mathrm{C}=0), 1611(\mathrm{C}=\mathrm{N}) .{ }^{1} \mathrm{H}$ NMR $\left(200 \mathrm{MHz}, \mathrm{CDCl}_{3}, \delta, \mathrm{ppm}\right): 9.7(\mathrm{~s}$, $1 \mathrm{H}, \mathrm{CONH}), 8.9\left(\mathrm{~s}, 2 \mathrm{H}, \mathrm{NH}_{2}\right), 7.2-7.5(\mathrm{~m}, 6 \mathrm{H}, \mathrm{Ar}-\mathrm{H}), 4.9(\mathrm{~s}, 2 \mathrm{H}$, $\mathrm{OCH}_{2}$ ). MS (EI, $\left.m / z(\%)\right): 217\left(\mathrm{M}^{+}, 22.54\right), 186(54.67), 145$ (100), 117 (50.54), 91 (2.25), 64 (5.75). Anal. calcd. for $\mathrm{C}_{11} \mathrm{H}_{11} \mathrm{~N}_{3} \mathrm{O}$ : C, 60.82; H, 5.10; N, 19.34. Found: C, 60.80; H, 5.08; N, $19.32 \%$.

\subsubsection{4-Amino-5-[(quinolin-8-yloxy)methyl]-4H-1,2,4- triazole-3-thiol (2)}

Carbon disulfide $(0.015 \mathrm{~mol})$ was added drop wise to a solution of hydrazide $1(0.01 \mathrm{~mol})$ in ethanol $(30 \mathrm{~mL})$ and potassium hydroxide $(0.015 \mathrm{~mol})$. The mixture was stirred at room temperature for $14 \mathrm{~h}$., dry diethyl ether $(20 \mathrm{~mL})$ was added and the separated solid was filtered off and washed with diethyl ether, the potassium salt was used for the next stage without further purification. 


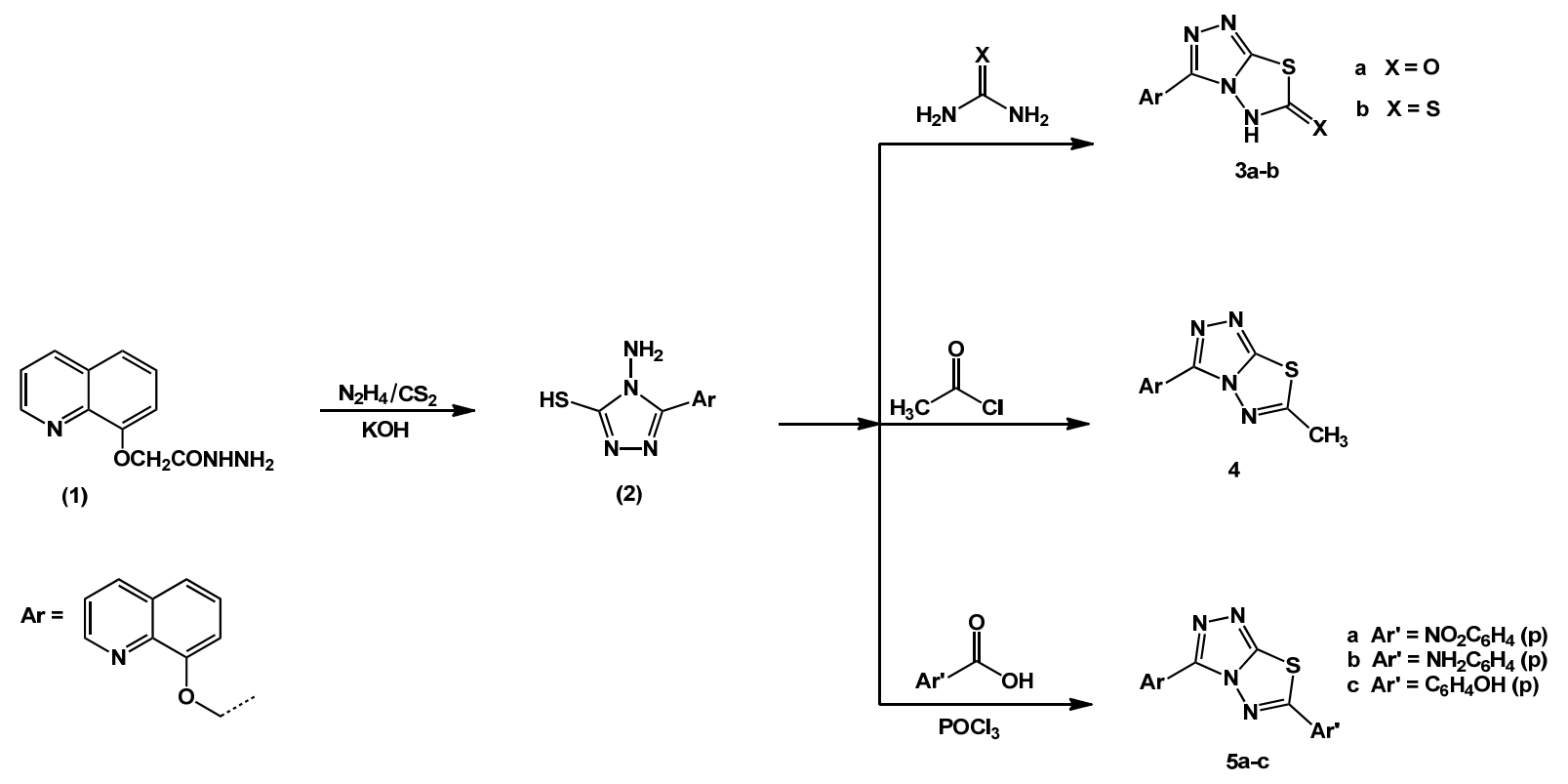

Scheme 1

Hydrazine hydrate $(0.02 \mathrm{~mol})$ was gradually added to the above potassium salt $(0.01 \mathrm{~mol})$ dissolved in water $(20 \mathrm{~mL})$ and all were refluxed with stirring for $4 \mathrm{~h}$. The reaction mixture was cooled and acidified with conc. HCl. A yellow solid separated out which was filtered, washed with water and purified by recrystallization from ethanol to afford triazole 2 (Scheme 1). Color: Yellow. Yield: 65\%. M.p.: 228-230 ${ }^{\circ}$ C. FT-IR (KBr, v, cm ${ }^{-1}$ ): 3402-3145 ( $\left.\mathrm{NH}_{2}\right), 2911\left(\mathrm{CH}_{2}\right), 2668(\mathrm{SH}), 1596$ (C=N). ${ }^{1} \mathrm{H}$ NMR $\left(200 \mathrm{MHz}, \mathrm{DMSO}-d_{6}, \delta, \mathrm{ppm}\right): 13.9$ (s, 2H, NH 2 ), 9.1(s, 1H, SH), 7.7-7.9 (m, 6H, Ar-H), $5.4\left(\mathrm{~s}, 2 \mathrm{H}, \mathrm{OCH}_{2}\right)$. MS (EI, $m / z(\%)): 273\left(\mathrm{M}^{+}, 24.82\right), 145$ (100), 117 (40.77), 91 (2.42), 64 (6.41). Anal. calcd. for $\mathrm{C}_{12} \mathrm{H}_{11} \mathrm{~N}_{5} \mathrm{OS}$ : C, 52.73; H, 4.06; N, 25.62. Found: C, 52.68; H, 4.02; N, 25.66\%.

\subsubsection{General procedure for preparation of 3a-b}

A mixture of triazole $2(0.01 \mathrm{~mol})$ and urea/thiourea $(0.015$ mol) was fused at $232{ }^{\circ} \mathrm{C}$ for $2 \mathrm{~h}$. After cooling, the solid was triturated with water and filtered off, washed and crystallized from ethanol.

Alternative method for synthesis of $\mathbf{3 b}$ : A mixture of an equimolar amount of compound 2 and $\mathrm{CS}_{2}(0.02 \mathrm{~mol})$ in DMF $(50 \mathrm{~mL})$ was refluxed for $6 \mathrm{~h}$. then it cooled and poured onto ice. The product was filtered off and crystallized from acetic acid (Scheme 1).

3-((Quinolin-8-yloxy)methyl)-[1,2,4]triazolo[3,4-b][1,3,4] thiadiazol-6(5H)-one (3a): Color: Pale yellow. Yield: $52 \%$. M.p.: 248-250 ㄷ. FT-IR (KBr, v, cm-1): 3409-3165 (NH), $2917\left(\mathrm{CH}_{2}\right)$, 1685 (C=0), 1618 (C=N), $1310 \mathrm{~cm}^{-1}$ (NCS). ${ }^{1} \mathrm{H}$ NMR $(200 \mathrm{MHz}$, DMSO- $\left.d_{6}, \delta, \mathrm{ppm}\right): 13.6(\mathrm{~s}, 1 \mathrm{H}, \mathrm{NH}), 8.6-7.2(\mathrm{~m}, 6 \mathrm{H}, \mathrm{Ar}-\mathrm{H}), 5.1(\mathrm{~s}$, $2 \mathrm{H}, \mathrm{OCH}_{2}$ ). MS (EI, $\left.m / z(\%)\right): 298\left(\mathrm{M}^{+1}, 25.05\right), 273$ (31.69), 257 (46.78), 157 (1.62), 145 (100). Anal. calcd. for $\mathrm{C}_{13} \mathrm{H}_{9} \mathrm{~N}_{5} \mathrm{O}_{2} \mathrm{~S}$ : C, 52.17; H, 3.03; N, 23.40. Found: C, 52.07; H, 2.99; N, 23.30\%.

3-((Quinolin-8-yloxy)methyl)-[1,2,4]triazolo[3,4-b][1,3,4] thiadiazol-6(5H)-thione (3b): Color: Yellow. Yield: 55\%. M.p.: 218-220 ${ }^{\circ}$ C. FT-IR (KBr, $\left.v, \mathrm{~cm}^{-1}\right): 3406(\mathrm{NH}), 2918\left(\mathrm{CH}_{2}\right), 1622$ $(\mathrm{C}=\mathrm{N}), 1375$ (NCS), 1279 (C=S). ${ }^{1} \mathrm{H}$ NMR (200 MHz, DMSO-d 6 , $\delta$, ppm): $8.8(\mathrm{~s}, 1 \mathrm{H}, \mathrm{NH}), 8.2-7.3(\mathrm{~m}, 6 \mathrm{H}, \mathrm{Ar}-\mathrm{H}), 5.2\left(\mathrm{~s}, 2 \mathrm{H}, \mathrm{OCH}_{2}\right)$. MS (EI, $m / z$ (\%)): 315 (M+, 15.87), 257 (3.85), 225 (12.50), 184 (3.13), 145 (100). Anal. calcd. for $\mathrm{C}_{13} \mathrm{H}_{9} \mathrm{~N}_{5} \mathrm{OS}_{2}: \mathrm{C}, 49.51 ; \mathrm{H}, 2.88$; N, 22.21. Found: C, 49.45; H, 2.75; N, 22.15\%.
2.2.4. 8-((6-Methyl-[1,2,4]triazolo[3,4-b][1,3,4]thiadiazol-3yl)methoxy)quinoline (4)

A mixture of triazole $2(0.01 \mathrm{~mol})$ and acetyl chloride $(0.01$ $\mathrm{mol}$ ) in dry pyridine ( $25 \mathrm{~mL})$ was heated for $2 \mathrm{~h}$ on steam bath. The reaction mixture was poured into crushed ice, the solid product obtained by filtration was purified by crystallization from ethanol (Scheme 1). Color: Yellow. Yield: 58\%. M.p.: 203$205{ }^{\circ} \mathrm{C}$. FT-IR $\left(\mathrm{KBr}, \mathrm{v}, \mathrm{cm}^{-1}\right): 2921\left(\mathrm{CH}_{2}\right), 1626(\mathrm{C}=\mathrm{N}), 1380$ (NCS). ${ }^{1} \mathrm{H}$ NMR $\left(200 \mathrm{MHz}\right.$, DMSO- $\left.d_{6}, \delta, \mathrm{ppm}\right): 8.2-7.5(\mathrm{~m}, 6 \mathrm{H}, \mathrm{Ar}-$ $\mathrm{H}), 5.6\left(\mathrm{~s}, 2 \mathrm{H}, \mathrm{OCH}_{2}\right), 2.4\left(\mathrm{~s}, 3 \mathrm{H}, \mathrm{CH}_{3}\right)$. MS (EI, $\left.m / z(\%)\right): 299$ $\left(\mathrm{M}^{+2}, 12.05\right), 258$ (8.89), 145(100), 56 (0.95). Anal. calcd. for $\mathrm{C}_{14} \mathrm{H}_{11} \mathrm{~N}_{5} \mathrm{OS}$ : C, 56.55; H, 3.73; N, 23.55. Found: C, 56.49; H, 3.68; N, $23.50 \%$.

\subsubsection{General procedure for preparation of $5 a-c$}

A mixture of triazole $2(0.01 \mathrm{~mol})$ and aromatic acids namely ( $p$-nitrobenzoic acid, $p$-aminobenzoic acid, $p$-hydroxy benzoic acid) $(0.01 \mathrm{~mol})$ in the presence of $\mathrm{POCl}_{3}(15 \mathrm{~mL})$ was refluxed for $3 \mathrm{~h}$. After removal of the excess of $\mathrm{POCl}_{3}$ under reduced pressure, the residue was added to crushed ice and stirred at room temperature for $1 \mathrm{~h}$. during this time the solution was gradually neutralized with $\mathrm{Na}_{2} \mathrm{CO}_{3}$, the solid product was filtered off, washed with water, dried and crystallized from DMF (Scheme 1).

8-((6-(4-Nitrophenyl)-[1,2,4]triazolo[3,4-b][1,3,4]thiadiazole-3-yl)methoxy)quinoline (5a): Color: Yellow. Yield: $72 \%$. M.p.: $150-152{ }^{\circ} \mathrm{C}$. FT-IR $\left(\mathrm{KBr}, v, \mathrm{~cm}^{-1}\right): 2924\left(\mathrm{CH}_{2}\right), 1606(\mathrm{C}=\mathrm{N})$, $1496\left(\mathrm{NO}_{2}\right), 1344$ (NCS). ${ }^{1} \mathrm{H}$ NMR $\left(200 \mathrm{MHz}\right.$, DMSO- $\left.d_{6}, \delta, \mathrm{ppm}\right)$ : 6.5-8.3 (m, 10H, Ar-H), $5.7\left(\mathrm{~s}, 2 \mathrm{H}, \mathrm{OCH}_{2}\right)$. MS (EI, $\left.m / z(\%)\right): 404$ (M+, 85.23), 257 (9.01), 225 (5.08), 145 (100), 117 (50.89), 91 (30.45), 64 (0.05). Anal. calcd. for $\mathrm{C}_{19} \mathrm{H}_{12} \mathrm{~N}_{6} \mathrm{O}_{3} \mathrm{~S}: \mathrm{C}, 56.43$; $\mathrm{H}$, 2.99; N, $20.78 \%$. Found: C, 56.35; H, 2.90; N, $20.68 \%$.

4-(3-((Quinolin-8-yloxy)methyl)-[1,2,4]triazolo[3,4-b][1,3,4] thiadiazol-6-yl)aniline (5b): Color: Yellow. Yield: 74\%. M.p.: 208-210 ${ }^{\circ}$ C. FT-IR (KBr, $\left.v, \mathrm{~cm}^{-1}\right): 3410\left(\mathrm{NH}_{2}\right), 2925\left(\mathrm{CH}_{2}\right), 1600$ $(\mathrm{C}=\mathrm{N}), 1316$ (NCS). ${ }^{1} \mathrm{H}$ NMR $\left(200 \mathrm{MHz}, \mathrm{DMSO}-d_{6}, \delta, \mathrm{ppm}\right): 11.9$ (s, $\left.2 \mathrm{H}, \mathrm{NH}_{2}\right), 6.9-8.2(\mathrm{~m}, 10 \mathrm{H}, \mathrm{Ar}-\mathrm{H}), 5.2\left(\mathrm{~s}, 2 \mathrm{H}, \mathrm{OCH}_{2}\right)$. MS (EI, $m / z$ (\%)): $375\left(\mathrm{M}^{+}, 10.89\right), 256$ (9.10), 145 (20.89), 117 (50.66), 91 (30.78), 64 (100). Anal. calcd. for $\mathrm{C}_{19} \mathrm{H}_{14} \mathrm{~N}_{6} \mathrm{OS}$ : C, 60.95; H, 3.77; N, 22.45. Found: C, 60.93; H, 3.75; N, 22.42\%. 


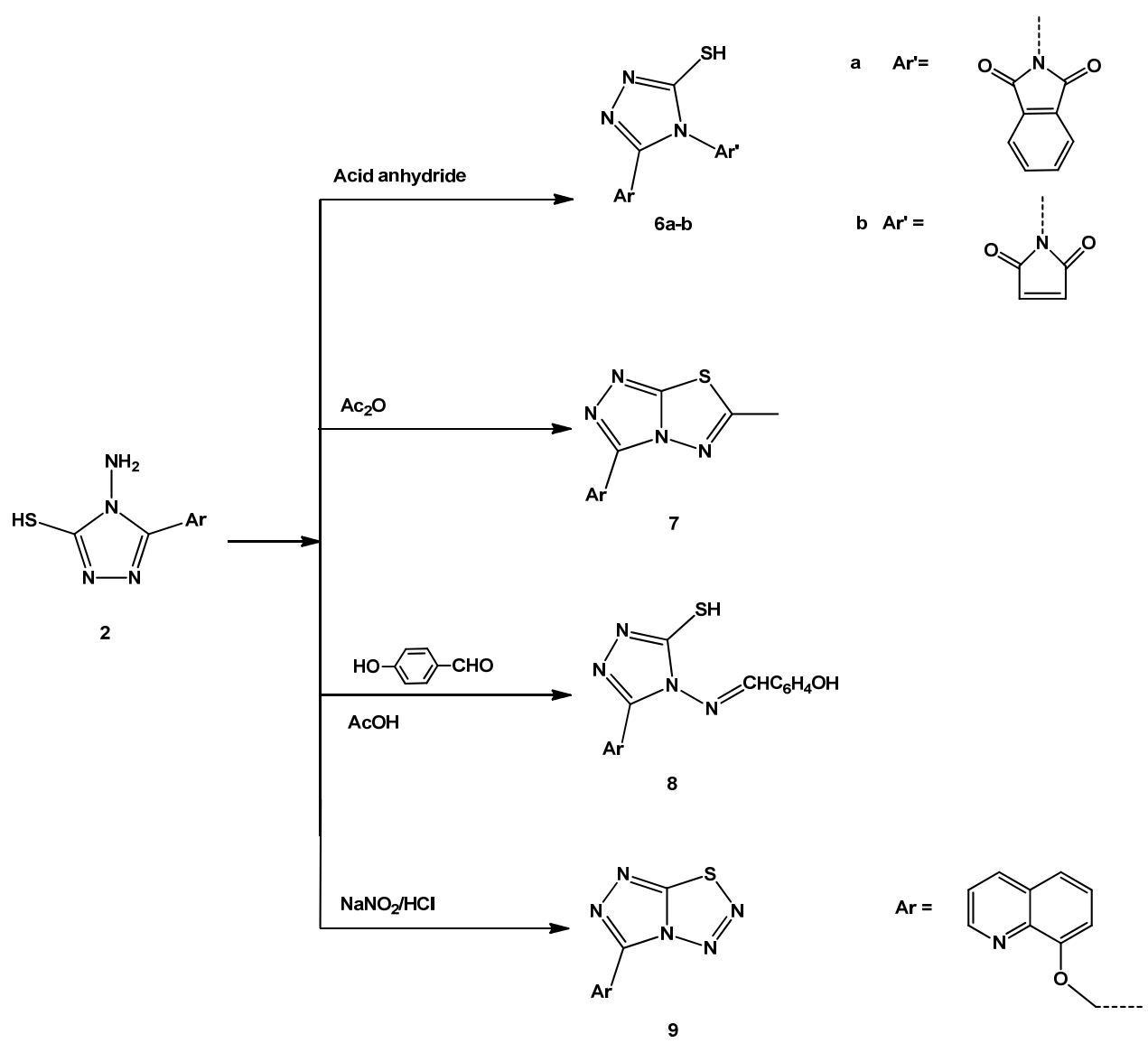

Scheme 2

4-(3-((Quinolin-8-yloxy)methyl)-[1,2,4]triazolo[3,4-b] [1,3,4]thiadiazol-6-yl)phenol (5c): Color: Yellow. Yield: 66\%. M.p.: 233-235 ${ }^{\circ} \mathrm{C}$. FT-IR (KBr, v, cm-1): $3387(\mathrm{OH}), 2925\left(\mathrm{CH}_{2}\right)$, $1598(\mathrm{C}=\mathrm{N}), 1377$ (NCS). ${ }^{1} \mathrm{H}$ NMR $\left(200 \mathrm{MHz}, \mathrm{DMSO}-d_{6}, \delta, \mathrm{ppm}\right)$ : $13.8(\mathrm{~s}, 1 \mathrm{H}, \mathrm{OH}), 7.4-8.3(\mathrm{~m}, 10 \mathrm{H}, \mathrm{Ar}-\mathrm{H}), 5.3\left(\mathrm{~s}, 2 \mathrm{H}, \mathrm{OCH}_{2}\right)$. Anal. calcd. for $\mathrm{C}_{19} \mathrm{H}_{13} \mathrm{~N}_{5} \mathrm{O}_{2} \mathrm{~S}$ : C, 60.79; $\mathrm{H}, 3.49 ; \mathrm{N}, 18.66$. Found: $\mathrm{C}$, $60.75 ; \mathrm{H}, 3.50 ; \mathrm{N}, 18.68 \%$.

\subsubsection{General procedure for preparation of $6 a, b$}

Triazole $2(0.01 \mathrm{~mol})$ was fused with phthalic anhydrid or maleic anhydride $(0.01 \mathrm{~mol})$ for $3 \mathrm{~h}$., the reaction mixture was cooled, triturated with water, the separated solid was filtered, washed with water and crystallized from ethanol (Scheme 2).

2-(3-Mercapto-5-((quinolin-8-yloxy)methyl)-4H-1,2,4triazol-4-yl)isoindoline-1,3-dione (6a): Color: Yellow. Yield: 75\%. M..p.: 126-128 ${ }^{\circ} \mathrm{C}$. FT-IR (KBr, v, cm-1): $2923\left(\mathrm{CH}_{2}\right), 2660$ (SH), 1749, 1649 (C=0), $1598(\mathrm{C}=\mathrm{N}) .{ }^{1} \mathrm{H}$ NMR $(200 \mathrm{MHz}$, DMSO$\left.d_{6}, \delta, \mathrm{ppm}\right): 8.8(\mathrm{~s}, 1 \mathrm{H}, \mathrm{SH}), 7.8-8.1(\mathrm{~m}, 10 \mathrm{H}, \mathrm{Ar}-\mathrm{H}), 4.9(\mathrm{~s}, 2 \mathrm{H}$, $\mathrm{OCH}_{2}$ ). MS (EI, $\left.m / z(\%)\right)$ : $403\left(\mathrm{M}^{+}, 2.85\right)$. Anal. calcd. for $\mathrm{C}_{20} \mathrm{H}_{13} \mathrm{~N}_{5} \mathrm{O}_{3} \mathrm{~S}$ : C, 59.55; H, 3.25; N, 17.36. Found: C, 59.50; $\mathrm{H}$, 3.35; N, $17.40 \%$.

1-(3-Mercapto-5-((quinolin-8-yloxy)methyl)-4H-1,2,4triazol-4-yl)-1H-pyrrole-2,5-dione (6b): Color: Pale yellow. Yield: 73\%. M.p.: 143-145 ${ }^{\circ} \mathrm{C}$. FT-IR $\left(\mathrm{KBr}, v, \mathrm{~cm}^{-1}\right): 2922\left(\mathrm{CH}_{2}\right)$, $2668(\mathrm{SH}), 1706,1680(\mathrm{C}=0), 1597(\mathrm{C}=\mathrm{N}) .{ }^{1} \mathrm{H}$ NMR $(200 \mathrm{MHz}$, DMSO- $\left.d_{6}, \delta, \mathrm{ppm}\right): 10.1(\mathrm{~s}, 1 \mathrm{H}, \mathrm{SH}), 8.6-7.7(\mathrm{~m}, 8 \mathrm{H}, \mathrm{Ar}-\mathrm{H}), 5.1(\mathrm{~s}$, $\left.2 \mathrm{H}, \mathrm{OCH}_{2}\right)$. MS (EI, $\left.m / z(\%)\right): 355\left(\mathrm{M}^{+2}, 4.77\right), 257$ (1.95), 158 (48.25), 145 (100), 117 (9.05), 91 (10.23), 64 (6.59). Anal. calcd. for $\mathrm{C}_{16} \mathrm{H}_{11} \mathrm{~N}_{5} \mathrm{O}_{3} \mathrm{~S}$ : C, 54.38; H, 3.14; N, 19.82. Found: C, 54.40; H, 3.12; N, 19.89\%.

\subsubsection{8-((6-Methyl- $[1,2,4]$ triazolo[3,4-b][1,3,4]thiadiazol-3- yl)methoxy quinoline (7)}

A solution of triazole $2(0.01 \mathrm{~mol})$ in acetic anhydride $(20$ $\mathrm{mL}$ ) was heated under reflux for $14 \mathrm{~h}$, after cooling; the reaction mixture was poured into ice. The obtained solid was crystallized from ethanol (Scheme 2). Color: Yellow. Yield: $57 \%$. M.p.: 200-202 ㄷ. FT-IR (KBr, v, cm-1): $2921\left(\mathrm{CH}_{2}\right), 1599$ (C=N), 1373 (NCS). ${ }^{1} \mathrm{H}$ NMR (200 MHz, DMSO- $\left.d_{6}, \delta, \mathrm{ppm}\right): 8.1-7.9(\mathrm{~m}$, $6 \mathrm{H}, \mathrm{Ar}-\mathrm{H}), 4.8\left(\mathrm{~s}, 2 \mathrm{H}, \mathrm{OCH}_{2}\right), 2.8\left(\mathrm{~s}, 3 \mathrm{H}, \mathrm{CH}_{3}\right)$. MS (EI, $\left.m / z(\%)\right)$ : 299 (M+2, 20.89), 259 (10.50), 179 (30.89), 92 (8.09), 56 (100). Anal. calcd. for $\mathrm{C}_{14} \mathrm{H}_{11} \mathrm{~N}_{5} \mathrm{OS}$ : C, 56.55; H, 3.73; N, 23.55. Found: C, $56.75 ; \mathrm{H}, 3.60 ; \mathrm{N}, 23.45 \%$.

2.2.8. 4-([3-Mercapto-5-(quinolin-8-yloxymethyl)-[1,2,4] triazol-4-ylimino]-methyl)-phenol (8)

A solution of triazole $2(0.01 \mathrm{~mol})$ in glacial acetic acid $(10$ $\mathrm{mL})$ reacted with $p$-hydroxy benzaldehyde $(0.01 \mathrm{~mol})$ under reflux for $1 \mathrm{~h}$., the reaction mixture was then cooled and the precipitated solid was filtered off, washed with water, dried and crystallized from ethanol (Scheme 2). Color: Yellow. Yield: 65\%. M.p.: 148-150 ㄷ. FT-IR (KBr, v, cm-1): $3416(\mathrm{OH}), 2923$ $\left(\mathrm{CH}_{2}\right), 2695(\mathrm{SH}), 1597(\mathrm{C}=\mathrm{N}) .{ }^{1} \mathrm{H}$ NMR $\left(200 \mathrm{MHz}, \mathrm{DMSO}-d_{6}, \delta\right.$, ppm): 13.8 (s, 1H, OH), 8.8 (s, 1H, SH), $8.3(\mathrm{~s}, 1 \mathrm{H}, \mathrm{CH}=\mathrm{N}), 7.4-8.1$ (m, 10H, Ar-H), 4.9 (s, 2H, OCH $)$. MS (EI, $m / z(\%)): 379\left(\mathrm{M}^{+2}\right.$, 36.28). Anal. calcd. for $\mathrm{C}_{19} \mathrm{H}_{15} \mathrm{~N}_{5} \mathrm{O}_{2} \mathrm{~S}: \mathrm{C}, 60.46 ; \mathrm{H}, 4.01 ; \mathrm{N}, 18.56$. Found: C, $60.55 ; \mathrm{H}, 4.10 ; \mathrm{N}, 18.65 \%$. 


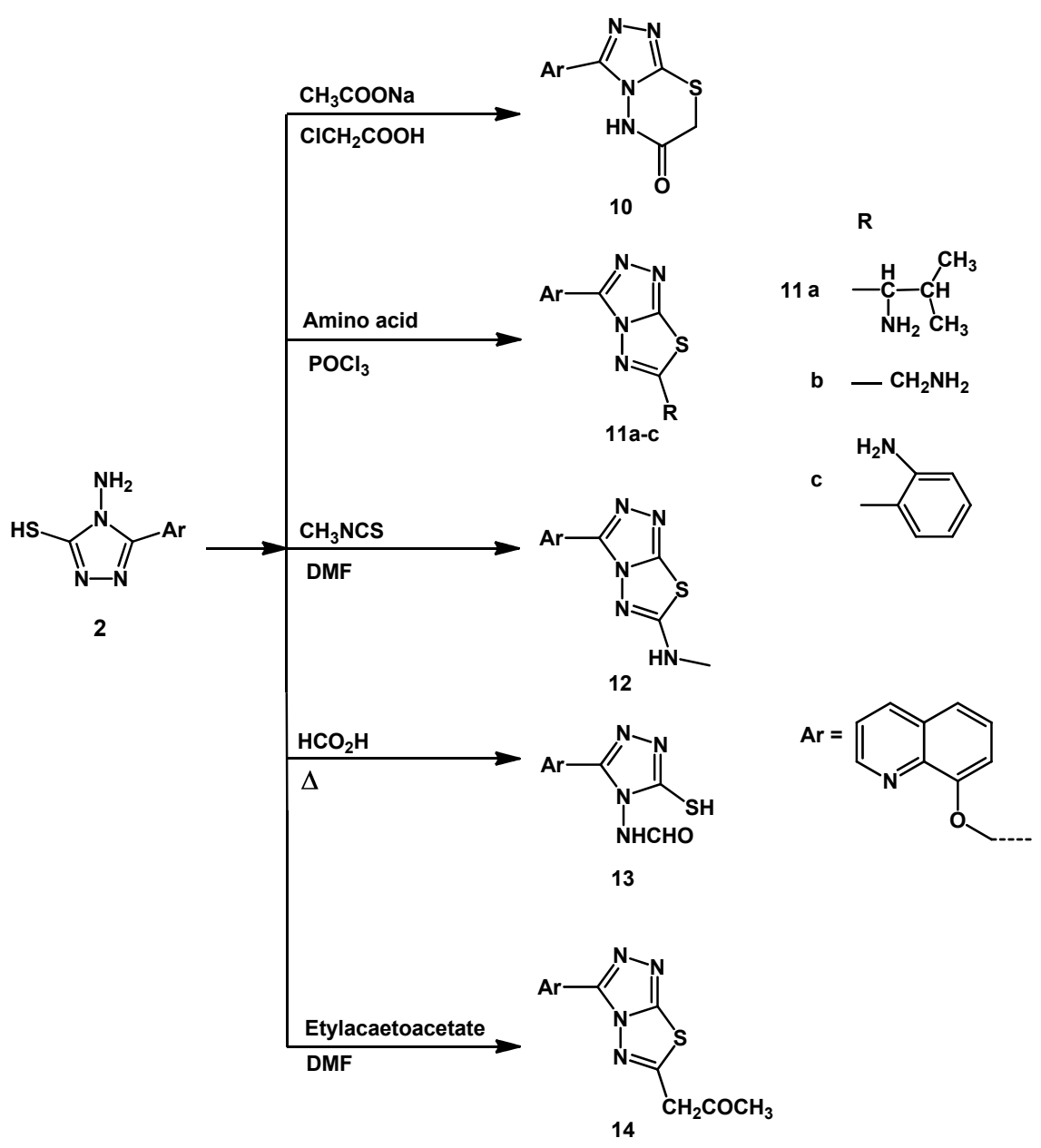

Scheme 3

\subsubsection{8-(([1,2,4]Triazolo[4,3-d][1,2,3,4]thiatriazol-6-yl) methoxy)quinoline (9)}

A solution of triazole $2(0.01 \mathrm{~mol})$ in $\mathrm{HCl}(50 \mathrm{~mL})$ was cooled to $0{ }^{\circ} \mathrm{C}$ and a cold solution of sodium nitrite $(0.01 \mathrm{~mol})$ in water $(10 \mathrm{~mL})$ was gradually added. The reaction mixture was kept at $0-5{ }^{\circ} \mathrm{C}$ with stirring for $2 \mathrm{~h}$, the mixture was left overnight and diluted with water where upon precipitation took place, the solid that precipitated was collected and crystallized from ethanol (Scheme 2). Color: Yellow. Yield: 50\%. M.p.: 128-130 ${ }^{\circ} \mathrm{C}$. FT-IR (KBr, v, cm-1): $2920\left(\mathrm{CH}_{2}\right), 1627$ (C=N), $1500(\mathrm{~N}=\mathrm{N}), 1385(\mathrm{NCS}) .{ }^{1} \mathrm{H}$ NMR $\left(200 \mathrm{MHz}, \mathrm{DMSO}-d_{6}, \delta, \mathrm{ppm}\right):$ 8.2-7.5 (m, 6H, Ar-H), 5.1 (s, 2H, OCH $)_{2}$. MS (EI, $\left.m / z(\%)\right): 284$ $\left(\mathrm{M}^{+}, 71.33\right), 257$ (1.4), 225 (12.50), 198 (17.48), 184 (3.13), 145 (100), 117 (85.72), 91 (18.54), 64 (61.80). Anal. calcd. for $\mathrm{C}_{12} \mathrm{H}_{8} \mathrm{~N}_{6} \mathrm{OS}$ : C, 50.70; H, 2.84; N, 29.56. Found: C, 50.65; H, 2.75; $\mathrm{N}, 29.77 \%$.

\subsubsection{3-((Quinolin-8-yloxy)methyl)-5H-[1,2,4]triazolo[3,4- b][1,3,4]thiadiazin-6-(7H)-one (10)}

To a solution of triazole $(0.01 \mathrm{~mol})$ in acetic acid $(50 \mathrm{~mL})$, chloroacetic acid $(0.01 \mathrm{~mol})$ and fused sodium acetate $(0.01$ $\mathrm{mol})$ were added. The reaction mixture was refluxed for $6 \mathrm{~h}$, the separated solid was filtered and recrystallized from ethanol (Scheme 3). Color: Pale yellow. Yield: 48\%. M.p.: 159-161 ${ }^{\circ} \mathrm{C}$. FT-IR (KBr, v, cm-1): $3423(\mathrm{NH}), 2922\left(\mathrm{CH}_{2}\right), 1659$ (C=0), 1635 $(\mathrm{C}=\mathrm{N}), 1390(\mathrm{NCS}) .{ }^{1} \mathrm{H}$ NMR $\left(200 \mathrm{MHz}, \mathrm{DMSO}-d_{6}, \delta, \mathrm{ppm}\right): 8.9$ (s,
$1 \mathrm{H}, \mathrm{NH}), 7.6-8.3(\mathrm{~m}, 6 \mathrm{H}, \mathrm{Ar}-\mathrm{H}), 5.4\left(\mathrm{~s}, 2 \mathrm{H}, \mathrm{CH}_{2}\right), 4.1(\mathrm{~s}, 2 \mathrm{H}$ $\left.\mathrm{OCH}_{2}\right)$. MS (EI, $\left.m / z(\%)\right)$ : $313\left(\mathrm{M}^{+2}, 3.56\right)$. Anal. calcd. for $\mathrm{C}_{14} \mathrm{H}_{11} \mathrm{~N}_{5} \mathrm{O}_{2} \mathrm{~S}$ : C, 53.66; $\mathrm{H}, 3.54 ; \mathrm{N}, 22.35$. Found: $\mathrm{C}, 53.75 ; \mathrm{H}_{4}$ $3.45 ; \mathrm{N}, 22.29 \%$.

\subsubsection{General procedure for preparation of 11a-c}

To a mixture of triazole $2(0.01 \mathrm{~mol})$ and different amino acids namely (anthranilic acid, glycine, and valine) $(0.01 \mathrm{~mol})$, $\mathrm{POCl}_{3}$ was added and the content was heated under reflux for $2 \mathrm{~h}$ on an oil bath. Excess of $\mathrm{POCl}_{3}$ was then removed and the residue was poured onto crushed ice and stirred well. These were then washed with $\mathrm{NaHCO}_{3}(5 \%)$ and the resulting solids were then, filtered, washed with water and crystallized from DMF (Scheme 3).

2-Methyl-1-[3-(quinolin-8-yloxymethyl)-[1,2,4]triazolo[3,4-b] [1,3,4]thiadiazol-6-yl]-propylamine (11a): Color: Yellow. Yield: 70\%. M.p.: 132-135 ${ }^{\circ}$ C. FT-IR (KBr, v, $\left.\mathrm{cm}^{-1}\right): 3417\left(\mathrm{NH}_{2}\right), 2962$ $\left(\mathrm{CH}_{2}\right), 1656(\mathrm{C}=\mathrm{N}), 1381$ (NCS). ${ }^{1 \mathrm{H}} \mathrm{NMR}\left(200 \mathrm{MHz}, \mathrm{DMSO}-d_{6}, \delta\right.$, ppm): $10.1\left(\mathrm{~s}, 2 \mathrm{H}, \mathrm{NH}_{2}\right), 7.3-8.1(\mathrm{~m}, 6 \mathrm{H}, \mathrm{Ar}-\mathrm{H}), 5.0\left(\mathrm{~s}, 2 \mathrm{H}, \mathrm{OCH}_{2}\right)$ $2.9(\mathrm{~m}, 2 \mathrm{H}, \mathrm{CH}), 1.6\left(\mathrm{~d}, 6 \mathrm{H}, 2 \mathrm{CH}_{3}\right)$. Anal. calcd. for $\mathrm{C}_{17} \mathrm{H}_{18} \mathrm{~N}_{6} \mathrm{OS}: \mathrm{C}$, $57.61 ; \mathrm{H}, 5.12 ; \mathrm{N}, 23.71$. Found: C, 57.50; H, 4.99; N, 23.80\%.

(3-((Quinolin-8-yloxy)methyl)-[1,2,4]triazolo[3,4-b][1,3,4] thiadiazol-6-yl)methanamine (11b): Color: Yellow. Yield: 62\%. M.p.: 188-190 ${ }^{\circ} \mathrm{C}$. FT-IR (KBr, v, cm-1 $): 3421\left(\mathrm{NH}_{2}\right), 2926\left(\mathrm{CH}_{2}\right)$, $1606(\mathrm{C}=\mathrm{N}), 1382$ (NCS). ${ }^{1} \mathrm{H}$ NMR $\left(200 \mathrm{MHz}\right.$, DMSO- $\left.d_{6}, \delta, \mathrm{ppm}\right)$ : 9.9 (s, 2H, NH $\mathrm{NH}_{2}, 7.6-8.3(\mathrm{~m}, 6 \mathrm{H}, \mathrm{Ar}-\mathrm{H}), 4.7$ (s, $\left.2 \mathrm{H}, \mathrm{OCH}_{2}\right), 2.9$ (s, $2 \mathrm{H}, \mathrm{CH}_{2}$ ). MS (EI, $\left.m / z(\%)\right): 313\left(\mathrm{M}^{+1}, 10.25\right), 160(0.05), 145$ 
(5.05), 91 (10.09), 64 (100). Anal. calcd. for $\mathrm{C}_{14} \mathrm{H}_{12} \mathrm{~N}_{6} \mathrm{OS}$ : C, 53.83; H, 3.87; N, 26.91. Found: C, 53.92; H, 3.77; N, 26.82\%.

2-(3-((Quinolin-8-yloxy)methyl)-[1,2,4]triazolo[3,4-b][1,3,4] thiadiazole-6-yl)benzenamine (11c): Color: Yellow. Yield: 68\%. M.p.: 98-100 ${ }^{\circ} \mathrm{C}$. FT-IR (KBr, v, cm$\left.{ }^{-1}\right): 3432\left(\mathrm{NH}_{2}\right), 2927\left(\mathrm{CH}_{2}\right)$, $1611(\mathrm{C}=\mathrm{N}), 1376(\mathrm{NCS}) .{ }^{1 \mathrm{H}}$ NMR $\left(200 \mathrm{MHz}, \mathrm{DMSO}-d_{6}, \delta, \mathrm{ppm}\right):$ $8.8\left(\mathrm{~s}, 2 \mathrm{H}, \mathrm{NH}_{2}\right), 6.6-8.3(\mathrm{~m}, 11 \mathrm{H}, \mathrm{Ar}-\mathrm{H}), 5.8\left(\mathrm{~s}, 2 \mathrm{H}, \mathrm{OCH}_{2}\right) . \mathrm{MS}$ (EI, $m / z(\%)): 374\left(\mathrm{M}^{+}, 4.27\right), 257$ (2.10), 145 (58.72), 117 (100), 91 (18.54), 64 (61.80). Anal. calcd. for $\mathrm{C}_{19} \mathrm{H}_{14} \mathrm{~N}_{6} \mathrm{OS}$ : C, 60.90; H, 3.77; N, 22.45. Found: C, 60.85; H, 3.65; N, 22.35\%.

\subsubsection{2. $N$-methyl-3-((quinolin-8-yloxy)methyl)-[1,2,4] triazolo[3,4-b][1,3,4]thiadiazol-6-amine (12)}

An equimolar amount of triazole $\mathbf{2}$ and methyl isothiocyanate $(0.01 \mathrm{~mol})$ in DMF $(30 \mathrm{~mL})$ was refluxed for $4 \mathrm{~h}$, cooled, and then poured onto ice. The product solid was filtered off and crystallized from ethanol (Scheme 3). Color: Yellow. Yield: 63\%. M.p.: 250-252 ${ }^{\circ} \mathrm{C}$. FT-IR (KBr, v, $\left.\mathrm{cm}^{-1}\right): 3323(\mathrm{NH})$, $2845\left(\mathrm{CH}_{2}\right), 1620$ (C=N), 1374 (NCS). ${ }^{1} \mathrm{H}$ NMR (200 MHz, DMSO$\left.d_{6}, \delta, \mathrm{ppm}\right): 9.1(\mathrm{~s}, 1 \mathrm{H}, \mathrm{NH}), 7.7-8.1(\mathrm{~m}, 6 \mathrm{H}, \mathrm{Ar}-\mathrm{H}), 4.9(\mathrm{~s}, 2 \mathrm{H}$, $\left.\mathrm{OCH}_{2}\right), 2.4\left(\mathrm{~s}, 3 \mathrm{H}, \mathrm{CH}_{3}\right)$. MS (EI, $\left.m / z(\%)\right): 313\left(\mathrm{M}^{+1,} 30.03\right), 257$ (20.43), 184 (0.73), 158 (7.44), 145 (100), 117 (35.78), 64 (5.05). Anal. calcd. for $\mathrm{C}_{14} \mathrm{H}_{12} \mathrm{~N}_{6} \mathrm{OS}$ : C, 53.83; H, 3.87; N, 26.91. Found: C, $53.75 ; \mathrm{H}, 3.95 ; \mathrm{N}, 26.85 \%$.

\subsubsection{N-(3-Mercapto-5-((quinolin-8-yloxy)methyl)-4H- 1,2,4-triazol-4-yl) formamide (13)}

Triazole $2(0.01 \mathrm{~mol})$ in formic acid $(10 \mathrm{~mL})$ was heated under reflux for $3 \mathrm{~h}$. The solid formed was collected by filtration and crystallized from ethanol (Scheme 3). Color: Yellow. Yield: $60 \%$. M.p.: 254-256 ${ }^{\circ} \mathrm{C}$. FT-IR $\left(\mathrm{KBr}, v, \mathrm{~cm}^{-1}\right): 3410$ (NH), $2857\left(\mathrm{CH}_{2}\right), 2729(\mathrm{SH}), 1713(\mathrm{C}=0), 1599(\mathrm{C}=\mathrm{N}) .{ }^{1} \mathrm{H}$ NMR (200 MHz, DMSO-d $6, \delta, \mathrm{ppm}): 12.3(\mathrm{~s}, 1 \mathrm{H}, \mathrm{SH}), 9.7$ (s, $1 \mathrm{H}, \mathrm{NH})$, $8.9(\mathrm{~s}, 1 \mathrm{H}, \mathrm{CHO}), 7.2-8.2(\mathrm{~m}, 6 \mathrm{H}, \mathrm{Ar}-\mathrm{H}), 4.8\left(\mathrm{~s}, 2 \mathrm{H}, \mathrm{OCH}_{2}\right)$. MS (EI, $m / z(\%)): 301\left(\mathrm{M}^{+}, 20.67\right), 257$ (10.78), 145 (100), 117 (45.56), 91 (30.54), 64 (35.89). Anal. calcd. for $\mathrm{C}_{13} \mathrm{H}_{11} \mathrm{~N}_{5} \mathrm{O}_{2} \mathrm{~S}: \mathrm{C}, 51.82 ; \mathrm{H}$, 3.68; N, 23.24. Found: C, 51.99; H, 3.65; N, 23.19\%.

\subsubsection{1-(3-((Quinolin-8-yloxy)methyl)-[1,2,4]triazolo[3,4- b][1,3,4]thiadiazol-6-yl)propan-2-one (14)}

A mixture of triazole $2(0.01 \mathrm{~mol})$, ethylacetoacetate $(0.02$ $\mathrm{mol})$ and triethylamine $(0.02 \mathrm{~mol})$ in DMF $(20 \mathrm{~mL})$ was heated under reflux for $12 \mathrm{~h}$. The precipitated solid was collected by filtration and crystallized from ethanol (Scheme 3). Color: Yellow. Yield: 60\%. M.p. 256-258 ${ }^{\circ}$ C. FT-IR (KBr, v, cm-1): 2919 $\left(\mathrm{CH}_{2}\right), 1679(\mathrm{C}=0), 1599(\mathrm{C}=\mathrm{N}), 1379(\mathrm{NCS}) .{ }^{1} \mathrm{H}$ NMR $(200 \mathrm{MHz}$, DMSO- $\left.d_{6}, \delta, \mathrm{ppm}\right): \delta$ ppm: 6.9-8.1 $(\mathrm{m}, 6 \mathrm{H}, \mathrm{Ar}-\mathrm{H}), 4.9(\mathrm{~s}, 2 \mathrm{H}$, $\left.\mathrm{OCH}_{2}\right), 4.2\left(\mathrm{~s}, 2 \mathrm{H}, \mathrm{CH}_{2}\right), 2.3\left(\mathrm{~s}, 3 \mathrm{H}, \mathrm{CH}_{3}\right)$. Anal. calcd. for $\mathrm{C}_{16} \mathrm{H}_{13} \mathrm{~N}_{5} \mathrm{O}_{2} \mathrm{~S}: \mathrm{C}, 56.63 ; \mathrm{H}, 3.86$; $\mathrm{N}, 20.64$. Found: $\mathrm{C}, 56.55 ; \mathrm{H}$, $3.80 ; \mathrm{N}, 20.59 \%$.

\subsection{Antimicrobial activity test}

The tested microorganisms were Gram-positive bacteria (Staphylococcus aureus) and Gram-negative bacteria (Escherichia coli). In addition, some fungi (Candida albicans and Aspergillus flavus) were also tested. On the other hand, Tetracycline and Amphotericin B were used as standard antibacterial and antifungal agents, respectively. The observed data on the antimicrobial activity of the compounds and the standard drugs are given in Table 1.

Agar diffusion method was used for the determination of the preliminary antibacterial and antifungal activity [31] and the results were recorded for each tested compound as the average diameter of inhibition zones ( $r$ ) of bacterial or fungal growth around the disks in millimeters at $100 \mu \mathrm{g}$ concentrations in dimethylsulfoxide.
The $\operatorname{Gr}(+)$ test organism and $\operatorname{Gr}(-)$ test organism were applied using 8 pore suspension of each test organism separately spreaded over a petri dish containing a solid nutrient agar medium of the following composition $(\mathrm{g} / \mathrm{L})$.

Beef extract, 3.0; tryptone, 5.0; Agar, 15.0; and distilled water, 1000.0. After immediately fresh inoculation of the tested organism, a well per each Petri dish was made, and the $0.1 \mathrm{~g}$ of the tested compound was filled the $6 \mathrm{~mm}$ in diameter well respectively then incubated at $37{ }^{\circ} \mathrm{C}$ for $48 \mathrm{~h}$, the diameter of the inhibition zone was measured in $\mathrm{mm}$. Also the antifungal activities were tested using spore suspension of each tested organism separately, spreaded over Petri dish containing solid Czapeks Dox agar of the following composition $(\mathrm{g} / \mathrm{L})$.

Sucrose, 30.0; $\mathrm{MgSO}_{4}, 0.5 ; \mathrm{KCl}, 0.5 ; \mathrm{FeSO}_{4}, 0.01 ; \mathrm{NaNO}_{3} 3.0$; $\mathrm{K}_{2} \mathrm{HPO}_{4}$ 1.0; Agar, 20.0; and distilled water, 1000.0. After immediately fresh inoculation of the test organism, a $6 \mathrm{~mm}$ well per each Petri dish was made, and the tested compounds were added to fill wells respectively, and then incubated at $28{ }^{\circ} \mathrm{C}$ for 7 days.

\section{Results and discussion}

\subsection{Synthesis}

Scheme 1-3 show the synthetic pathways to prepare the target compounds 2-14. The starting material 4-amino-5[(quinolin-8-yloxy) methyl]-4H-1,2,4-triazole-3-thiole (2) was synthesized from the reaction of acid hydrazide 1 [30] with carbon disulfide in ethanol containing potassium hydroxide followed by treatment with hydrazine hydrate. The structure of triazole 2 was elucidated by ${ }^{1} \mathrm{H}$ NMR spectrum that showed signals at $\delta 13.9$ and $10.5 \mathrm{ppm}$ corresponding to $\mathrm{NH}_{2}$ and $\mathrm{SH}$ protons respectively. Also, IR spectrum displays absorption bands at 3263-3145 and $2668 \mathrm{~cm}^{-1}$ due to $\mathrm{NH}_{2}$ and $\mathrm{SH}$ absorption (Scheme 1).

Triazole $\mathbf{2}$ with the reactive amino and thio groups proved to be versatile key precursor for constructing wide range of fused triazole derivatives. Thus, the reactivity of triazole $\mathbf{2}$ towards variety of chemical reagents with the aim of synthesis of fused heterocyclic systems of expected biological activity was investigated. The reaction of compound 2 with urea or thiourea afforded $[1,2,4]$-triazolo-[3,4- $b][1,3,4]$-thiadiazoles 3a and $\mathbf{3 b}$ respectively. Compound $\mathbf{3 b}$ was prepared also from the reaction of 1,2,4-triazole 2 with carbon disulfide in DMF. The structures of the products were assigned on the basis of their spectral data and elemental analysis. Treatment of triazole 2 with acetyl chloride in dry pyridine afforded product 4 . On the other hand, reaction of triazole $\mathbf{2}$ with aromatic acids namely ( $p$-nitrobenzoic acid, $p$-aminobenzoic acid and $p$-hydroxy benzoic acid in the presence of $\mathrm{POCl}_{3}$ gave 5a-c respectively (Scheme 1).

Furthermore, heating of triazole $\mathbf{2}$ with acid anhydride namely phthalic anhydride or maleic anhydride afforded triazole derivatives $\mathbf{6 a}$ and $\mathbf{6 b}$ respectively where as refluxing of triazole $\mathbf{2}$ in acetic anhydride due to ring closure to afford 8( (6-methyl-[1,2,4]triazolo[3,4-b][1,3,4] thiadiazole 7. However, triazole derivative 8 can be synthesized from reaction of triazole $\mathbf{2}$ with $p$-hydroxybenzaldhyde in the glacial acetic acid. Treatment of triazole $\mathbf{2}$ with sodium nitrite gave product $\mathbf{9}$ (Scheme 2).

Furthermore, the reaction of triazole 2 with chloroacetic acid in the presence of sodium acetate furnished triazolo[3,4b] $[1,3,4]$ thiadiazin derivative $\mathbf{1 0}$.

The reactivity of triazole 2 towards amino acids to give triazolo[3,4- $b][1,3,4]$ thiadiazole derivatives was investigated. Thus, the reaction of triazole $\mathbf{2}$ with valine, glycine and anthranilic acid in the presence of $\mathrm{POCl}_{3}$ due to formation of triazoles 11a-c, respectively (Scheme 3 ). The structures of the products were confirmed on the basis of their spectral data. 
Table 1. Antimicrobial activities of the synthesized compound *.

\begin{tabular}{|c|c|c|c|c|}
\hline Compound No. & Escherichia coli & Staphylococcus aureus & Aspergillus flavus & Candida albicans \\
\hline $3 a$ & - & - & - & - \\
\hline $3 b$ & 21 & 20 & - & 12 \\
\hline 4 & 14 & 12 & - & 12 \\
\hline $5 a$ & - & - & - & 11 \\
\hline $5 b$ & 12 & 11 & - & - \\
\hline $5 c$ & 13 & 11 & - & 12 \\
\hline $6 a$ & 17 & 17 & 12 & 12 \\
\hline $6 b$ & 21 & 20 & 11 & 12 \\
\hline 7 & - & - & - & - \\
\hline 8 & 14 & 14 & - & 13 \\
\hline 9 & 14 & 12 & - & - \\
\hline 10 & 14 & 12 & - & - \\
\hline $11 \mathrm{a}$ & 15 & 13 & - & - \\
\hline $11 \mathrm{~b}$ & 13 & 10 & - & - \\
\hline $11 \mathrm{c}$ & 15 & 11 & - & - \\
\hline 12 & 15 & 13 & - & 13 \\
\hline Tetracycline & 24 & 22 & - & - \\
\hline Amphotericin B & - & - & 13 & 14 \\
\hline
\end{tabular}

observed, i.e. compound not active; $(r)>10 \mathrm{~mm}$, slightly active; $(\mathrm{r})>13 \mathrm{~mm}$, moderately active and $(\mathrm{r})>20 \mathrm{~mm}$, high active.

Reaction of compound $\mathbf{2}$ with methyl isothiocyanate in the presence of DMF gave heterocycle 12. On the other hand, heating of triazole $\mathbf{2}$ with formic acid afforded triazole 13. When triazole 2 was allowed to react with 1,3-dicarbonyl compounds like ethylacetoacetate, triazole $\mathbf{1 4}$ was formed (Scheme 3).

\subsection{Antimicrobial activity}

1,2,4-Triazole derivatives were reported to have biological activity. In this study, some of the synthesized compounds containing 1,2,4-triazole moiety appear to be promising as potential bio responses which increase their importance towards application in pharmacological, industrial and agriculture fields. Therefore, their effectiveness against a number of microorganisms was tested (Table 1).

The results revealed that majority of the synthesized compounds showed varying degrees of inhibition against the tested microorganisms. In general, the inhibitory activity against the Gram-positive bacteria and Gram-negative bacteria was higher than that of the tested fungi. The tested compounds showed moderate (6a, 11 and 12 ) to high (3b and 6b) inhibitory effect towards tested bacteria (Table 1). Introduction of thiadiazolethione and pyrrolidindione moiety to triazole ring to give mixed heterocyclic systems $\mathbf{3 b}$ and $\mathbf{6 b}$ enhance antibacterial activities when compared with Tetracycline. It has been observed through the results in Table 1 that $\mathbf{6 a}$ and $\mathbf{6 b}$ derivatives showed both antifungal and antibacterial activities towards the tested organisms. On the other hand, triazoles 8 and 12 showed moderate activity towards the most tested microorganisms, while it is inactive towards Aspergillus flavus. In summary, the presence of thiadiazole moiety attached to fused triazole system enhances the inhibition zones of the tested microorganisms.

\section{Conclusion}

In the present paper, we have described the synthesis of 1,2,4-triazole derivatives fused to five and six membered rings from 4-amino-5-[(quinolin-8-yloxy)methyl]-4H-1,2,4-triazole3-thiole (2). It was observed that some of the synthesized compounds behave as efficient antibacterial and antifungal agents when compared with standard drugs. The structure of all derivatives was confirmed and characterized by elemental analysis and spectroscopic studies.

\section{Acknowledgement}

The authors wish to thank Chemistry Department, Faculty of Science, Benha University, Egypt for providing required chemicals to complete this work.

\section{References}

[1]. Karabasanagouda, T.; Adhikari, A. V.; Shetty, N. S. Eur. J. Med. Chem. 2007, 42, 521-525.

[2]. Mudasir, R. B.; Abdulrauf, A. Ind. J. Chem. B 2009, 43, 97-102.

[3]. Nirmala, K.; Parimala, S. Ind. J. Heterocycl. Chem. 2008, 17, 331-334.

[4]. Sztanke, K.; Tuzimski, T.; Rzymowska, J.; Pasternak, K.; Kanadefer, S. M. Eur. J. Med. Chem. 2008, 43(2), 404-409.

[5]. Harendra, S.; Manoj, K. S. Ind. J. Chem. B 2001, 40, 159-162.

[6]. Zareef, M.; Iqbal, R.; Mirza, B.; Khalid, M. K.; Manan, A.; Asim, F.; Khan, S.W. Arkivoc 2008, 2, 141-152.

[7]. Almasirad, A.; Tabatabai, S. A.; Faizi, M; Kebria, A; Mehrabi, N Dalavand, A; Shafiee, A. Bioorg. Med. Chem. 2004, 14, 6057-6061.

[8]. Tozkoparan, B.; Kupeli, E.; Yesilada, E.; Isil, S.; Ozalp, M.; Ertan, M. Arznei. Forschung 2005, 55(9), 533-538.

[9]. Shashikant, V. B.; Kailash, G. B.; Aniket, P. S.; Ajit , A.; Chetan , V. K.; Sudarshan, C. D. Pharmacologyonline 2008, 2, 572-587.

[10]. Giorgio, R.; Giancarlo, G.; Mario, D. B.; Daniela, P.; Vigilio, B.; Massimiliano, T.; Simona, B.; Elisabetta, B. Eur. J. Med. 2008, 43, 16651680.

[11]. Menendez, C.; Chollet, A.; Rodriguez, F.; Inard, C.; Pasca, M.; Lherbet C.; Baltas, M. Eur. J. Med. Chem. 2012, 52, 275-283

[12]. Kucukguzel, I.; Guniz-Kucukguzel, S.; Rollas, S.; Otuk-Sanis, G.; Ozdemir, O.; Bayrak, I.; Altug, T.; Stables, J. II Farmaco 2004, 59(11), 893-901.

[13]. Nadeem, S.; Mash, A.; Waqua, R. A. Acta Pharm. 2008, 58, 445-454.

[14]. Jing, C.; Xian-Yu, S.; Kyu-Yun, C.; Jin-Seok, L.; Mi-Sun, S.; Zhe-Shan, Q. B. Med. Chem. 2007, 15, 6775-6781.

[15]. Mohamd, A.; Harish, K. Ind. J. Chem. 2007, 46, 1014-1019.

[16]. Padmavathi, V.; Sudhakar, G. R.; Padmaja, A.; Kondaiah, P.; Ali, S. Eur. J. Med. 2009, 44, 2106-2112.

[17]. Kane, J. M.; Dubley, M. W.; Sorensen, S. M.; Miller, F. P. J. Med. Chem. 1988, 31, 1253-1255.

[18]. Athanasia, V.; Theodora, S. P.; Andrew, T.; Anna, T. K.; Alexandra, V. II Farmaco 1998, 53, 320-326.

[19]. Bing, C.; Xuhong, Q.; Song, C.; Haidong , L.; Gonghua , S. Arkivoc 2003, 2, 141-145.

[20]. Gamal, A. I.; Omar, M. A.; Gamal El-Din, A. A.; Abou-Rahma, M. F. Radwan, M. Eur. J. Med. 2009, 45, 1-8.

[21]. Michael, S. M.; Janet, S.; Michael, M.; Iwan, G.; Brenda, M.; Donald, S. Eur. J. Med. 2001, 36, 31-42.

[22]. Ouyang X.; Piatnitski, E. L.; Pattaropong, V.; Chen, X.; He, H. Y. Kiselyov, A. S.; Velankar, A.; Kawakami, J.; Labelle, M.; Smith, L.; Lohman, J.; Lee, S. P.; Malikzay, A.; Fleming, J.; Gerlak, J.; Wang, Y.; Rosler, R. L.; Zhou, K.; Mitelman, S.; Camara, M.; Surguladze, D.; Doody, J. F.; Tuma, M. C.Bioorg. Med. Chem. Lett. 2006, 16, 1191-1196.

[23]. Priyadarsini, R.; Vijayaraj, T. K.; Ravi, C.; Praba, M. Ind. J. Heterocycl. Chem. 2004, 20, 165-166.

[24]. Udupi, R. H.; Bhat, A. R. Ind. J. Heterocycl. Chem. 1996, 6, 41-45.

[25]. Dhanabal, T.; Sangeetha, R.; Mohan, P. S. Tetrahedron 2006, 62, 62586262.

[26]. Arzel, E.; Rocca, P.; Grellier, P.; Labaeid, M.; Frappier, F.; Gueritte, F.; Gaspard, C.; Marsais, F.; Godard, A.; Queguiner, G. J. Med. Chem. 2001 44, 949-954.

[27]. Aly, A. A.; Behalo, M. S. J. Chem. Res. 2010, 34(10), 571-575.

[28]. Behalo, M. S.; Aly, A. A. Eur. J. Chem. 2011, 2(3), 295-299.

[29]. Issac, Y. A.; El-Karim, I. G.; Donia, S. G.; Behalo, M. S. Sulf. Lett. 2002, 25(4), 183-190.

[30]. Mohd, A.; Rajesh, A. Ind. J. Heterocycl. Chem. 1998, 7, 225-228

[31]. Leifert, C.; Chidbouree, S.; Hampson, S.; Workman, S.; Sigee, D.; Epton, H. A. S.; Harbour, A. J. Appl. Bacteriol. 1995, 78, 97-102. 